\title{
8. RADIO COUNTERPARTS OF X-RAY SOURCES AND X-RAY COUNTERPARTS OF RADIO STARS
}

\author{
R. M. HJELLMING \\ National Radio Astronomy Observatory*, Green Bank, West Virginia, U.S.A.
}

\begin{abstract}
Some of the implications of the data on radio counterparts of X-ray sources are discussed. Data on the radio counterpart of Cyg X-3 is compared with the data on the radio star $\beta$ Persei (Algol), to show that similar radio emission mechanisms are involved. The data for such radio stars not yet known as X-ray sources imply that such objects should be transient X-ray sources.
\end{abstract}

The principal data concerning the radio counterparts of X-ray sources have just been reviewed by Braes and Miley (this volume, p. 86). This paper will discuss some of the implications of the radio data and a class of radio stars which should be transient $\mathrm{X}$-ray sources, although they have not yet been detected as such. Up until the recent detection of Cyg X-3 (Braes and Miley, 1972b; Hjellming et al., 1972b), the latter conclusion was largely theoretical; however, the newly observed radio properties of Cyg X-3 make it a virtual certainty that the radio binary stars (like $\beta$ Persei and $\beta$ Lyrae) and at least some of the $\mathrm{X}$-ray stars involve the same phenomena at radio wavelengths.

Let us begin by referring to Table I, which summarizes the properties of the known radio counterparts of compact galactic $\mathrm{X}$-ray sources. The radio variability and flux

TABLE I

$\mathrm{X}$-ray stars with known radio counterparts

Radio characteristics

\begin{tabular}{|c|c|c|c|c|c|c|}
\hline \multirow[t]{2}{*}{ Name } & \multirow[t]{2}{*}{ Spectrum } & \multirow{2}{*}{$\begin{array}{l}\text { Factor of } \\
\text { variability }\end{array}$} & \multicolumn{3}{|c|}{ Flux density (f.u.) } & \multirow[t]{2}{*}{ Optical identification } \\
\hline & & & Min. & Max. & Freq. & \\
\hline Sco X-1 & $\begin{array}{l}\text { Mostly non-thermal } \\
\text { some thermal }\end{array}$ & At least 60 & $<0.004$ & 0.25 & 2695 & $\begin{array}{l}\text { Peculiar blue star } \\
\text { variable in emission lines } \\
\text { and optical continuum }\end{array}$ \\
\hline$G \times 17+2$ & Non-thermal & At least 2 & $<0.005$ & 0.02 & 2695 & $?$ \\
\hline GX9+1 & Non-thermal & At least 1.5 & $<0.005$ & 0.008 & 2695 & ? \\
\hline Cyg X-1 & $\begin{array}{l}\text { Non-thermal } \\
\text { and thermal }\end{array}$ & $\begin{array}{l}\text { Off then on, } \\
\text { by at least } \\
\text { factor of } 3 \text {, } \\
\text { steady since }\end{array}$ & $<0.005$ & $\begin{array}{l}0.015 \\
0.021\end{array}$ & $\begin{array}{l}8085 \\
1415\end{array}$ & $\begin{array}{l}\text { BO Ib spectroscopic } \\
\text { binary peculiar emission } \\
\text { lines }\end{array}$ \\
\hline Cyg X-3 & $\begin{array}{l}\text { Mostly thermal } \\
\text { some non-thermal }\end{array}$ & At least 10 & $\begin{array}{l}0.02 \\
0.1 \\
0.1\end{array}$ & $\begin{array}{l}0.23 \\
0.32 \\
0.52\end{array}$ & $\begin{array}{l}1415 \\
2695 \\
8085\end{array}$ & \\
\hline
\end{tabular}

* The National Radio Astronomy Observatory is operated by Associated Universities, Inc. under contract with the U.S. National Science Foundation. 
density levels are well known, except for the newest and strongest entry, Cyg X-3. The first three sources are similar in that they are dominated by variable non-thermal radio emission; we can say that this is characteristic of radio counterparts of the Sco X-1 type.

\section{Sco X-1}

Only Sco X-1, of the sources dominated by non-thermal radio emission, is strong enough to give much information. Back in the days when there was one measurement (Andrew and Purton, 1968) of a radio flux at one frequency at one time and another measurement (Ables, 1969) at another frequency at another time, there were a number of theoretical papers interpreting the radio spectrum of Sco X-1. Since it has been proven to be a triple radio source (Hjellming and Wade, 1971a; Wade and Hjellming, 1971b; Braes and Miley, 1971a), with a central component sitting on the star which is presumably the X-ray source, that is variable by at least a factor of 60 , there have been, to my knowledge, no theoretical papers interpreting Sco $\mathrm{X}-1$. To the radio astronomer the potentially most interesting aspect of Sco X-1 is the double source surrounding the X-ray star and the central component, as shown in Figure 1. This is,

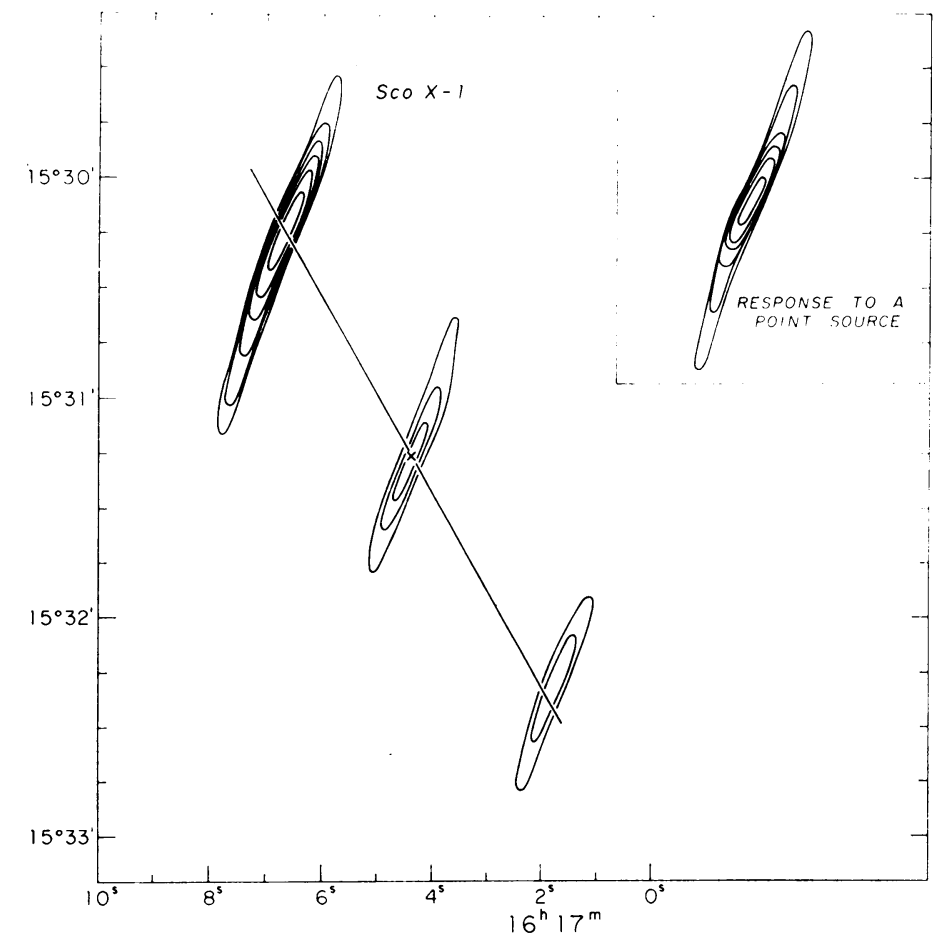

Fig. 1. A map of the Sco X-1 field derived from $2695 \mathrm{MHz}$ observations with the NRAO interferometer during February, March, and April 1971. The position of the X-ray star is shown by a $\times$. The diagonal line through the component sources is at position angle $29^{\circ}$. The coordinate grid is for epoch 1950.0. The synthesized beam image of a point source is shown in the upper right hand corner. 
of course, because of the common but yet largely unexplained presence of double radio sources symmetrically placed about interesting galaxies. Evidence is accumulating, but is not yet completely conclusive, that the NE companion has a variable radio flux density; if and when the evidence becomes conclusive it will be a virtual certainty that the radio companions are associated with Sco X-1. Because of the nonthermal spectra (Hjellming and Wade, 1971a; Wade and Hjellming, 1971b; Braes and Miley, 1971a) of the companions and the association with the X-ray star, it is hard to avoid the conclusion that the most likely explanation will identify the companions with complexes of coupled plasma and magnetic fields which are blasted with cosmic ray electrons from the X-ray star. One cannot say whether the two companions represent the only regions with the magnetic fields needed to produce a radio source, or whether a more extensive shell is being bombarded by two highly beamed streams of cosmic ray electrons. The resolution of these questions may have enormous significance for the physics of double radio sources.

Very little can be said about the nature of the variable central component. Because of its dominantly non-thermal nature, one tends to think first of a variably supplied synchotron spectrum. Unfortunately, the spectrum flattening proceeds while the flare is still clearly on the rise. One must conclude that the relative supply of higher and lower energy cosmic ray electrons alters during the course of a flare in the sense of the relative proportion at higher energies increasing. The only other suggestion of reproducible behavior while flaring is the suggestion of a repeated step-like behavior at both frequencies (Wade and Hjellming, 1971b).

There is also clear indication of the rare presence of a thermal component in Sco X-1. Two successive days in February 1970 (Wade and Hjellming, 1971b), just before the great flare of February 26, 1970, showed a stable radio source with a perfectly flat spectrum.

\section{Cyg X-1}

The radio data for Cyg X-1 has been singularly uninteresting in its own right - except for the unusual fact that during March-April 1971, the radio source jumped from below the detection limit to its present apparently steady state (Braes and Miley, 1971 b; Hjellming and Wade, 1971b; Hjellming et al., 1971; Braes and Miley, 1972a). The enormous significance of this event derives from the recently announced UHURU result (see review paper by Tananbaum, this volume, p. 9) that the time-averaged $\mathrm{X}$-ray flux underwent a change from one steady level to another during this time. Little can be said about the current Cyg X-1 radio source except that it may have both a thermal and a non-thermal component (Hjellming et al., 1971) and it has remained steady now for a full year.

The most important contribution of the radio counterpart of Cyg X-1 is its role in the identification of Cyg X-1 with a spectroscopic binary with a 9th magnitude BOIb component.

Since short time scale variability of a radio source near an X-ray source has been adopted as a necessary criterion for telling a radio counterpart of an $\mathrm{X}$-ray source 
from the very common and relatively steady background sources, clear identification of a Cyg X-1 type radio counterpart will always be difficult. We have no idea how common discontinuities in radio and $X$-ray properties might be. Cases where a steady radio source sits within an $\mathrm{X}$-ray error box will allow identification of a radio counterpart of the Cyg X-1 type only if it is identifiable with an obviously peculiar star. However, one must wrestle with the dilemma of whether there are radio stars which behave the same way but are not X-ray sources, as we will now discuss.

\section{Radio Binary Stars}

There are three radio binary stars that are not yet known to be $\mathrm{X}$-ray sources: $\beta$ Persei (Algol), $\beta$ Lyrae (Wade and Hjellming, 1972; Hjellming et al., 1972a), and Antares B (Wade and Hjellming, 1971a; Hjellming and Wade, 1971c). They are all variable on time scales of hours, as are the Sco X-1 type radio counterparts, but their dominant characteristic is that they appear like variable thermal bremsstrahlung sources when flaring. The recently detected radio counterpart to Cyg X-3 (Braes and Miley, 1972b; Hjellming et al., 1972b) shows radio characteristics virtually identical to the radio binary stars, particularly $\beta$ Persei (Algol).

\section{4. $\beta$ Persei (Algol)}

Because it is the strongest of the radio binary stars, $\beta$ Persei (Algol) can be taken as the prototype of the class. It is noteworthy that the discovery of the Cyg X-3 radio counterpart, which is very similar to $\beta$ Persei, occurred a week after a paper was written (Hjellming, 1972) arguing that the simplest interpretation of the $\beta$ Persei radio data required it to be a transient $X$-ray source. Let us therefore summarize the arguments (Hjellming, 1972) that lead to this result.

With the exception of a couple of hours on January 22-23, 1972, the radio spectrum of $\beta$ Persei has always appeared thermal. Initially it was believed (Wade and Hjellming, 1972; Hjellming et al., 1972a) that a thermal interpretation was unlikely; but now there are compelling reasons for just the opposite view. The radio flux density for a high temperature thermal source can be written (Hjellming, 1972) as

$$
S_{v} \cong \frac{2 k T_{e}}{\hat{\lambda}^{2}} \Omega_{s}\left[1-\exp \left(-0.28 E T_{e}^{-1.45} v_{\mathrm{GHz}}^{-2.05}\right)\right]
$$

where $T_{e}=$ electron temperature, $\Omega_{s}=$ solid angle subtended by the emitting source, $E=$ emission measure, $k=$ the Boltzmann constant, $v=$ the frequency, and $\lambda=$ wavelength. Measurement of $S_{v}$ at two frequencies, when the argument in the exponential (optical depth) is large enough, allows empirical determination of $T_{e} \Omega_{s}$ and $E T_{e}^{-1.45}$. Knowing the distance of the source, we can then empirically determine $T_{e} D^{2}$ and $D N_{e}^{2} T_{e}^{-1.45}$, where $D$ is the source diameter. When the source is a point source at $8085 \mathrm{MHz}$ for the NRAO interferometer, this means the angular diameter is less than 0 .'5, which puts an upper limit on $D$. This, with knowledge of $T_{e} D^{2}$ and $D N_{e}^{2} T_{e}^{-1.45}$, 
puts lower limits on $T_{e}$ and $N_{e}$. In addition, the fact that radio waves cannot pass through too dense a plasma puts an upper limit on $N_{e}$, hence an upper limit on $T_{e}$ and lower limits on $D$ and the angular diameter $\theta$. For $\beta$ Persei, with a distance of $25 \mathrm{pc}$, this means:

$$
\begin{array}{ll}
\theta<0.5 & N_{e}<9 \times 10^{10} \mathrm{~cm}^{-3} \\
T_{e}>2.5 \times 10^{5} \mathrm{~K} & T_{e}<2 \times 10^{9} \mathrm{~K} \\
D<13 \mathrm{AU} & D>0.1 \mathrm{AU} \\
N_{e}>6 \times 10^{6} \mathrm{~cm}^{-3} & \theta>0.004
\end{array}
$$

The lower limit on $T_{e}$ is immediately interesting because at temperatures only slightly above that limit, $\beta$ Persei would be a soft X-ray source. There are two arguments indicating that when flaring most strongly, $\beta$ Persei is closer to the upper limit on $T_{e}$. First, the only way the optical emission lines could be as weak as observed would be if the gas is much hotter than a normal $10^{4}-10^{5} \mathrm{~K}$ plasma. Secondly, we can estimate the order of magnitude of all the parameters of the thermal source when at its strongest. As is well known for observations of the solar chromosphere and corona, radio observations of a plasma in the gravitational potential well of a star tend to see radiation from different density levels at different frequencies. It is a well known rule of thumb that such radio emission, at a frequency $v$, occurs dominantly from regions just out side the level where $v$ equals the plasma frequency. We can therefore argue that, because the $\beta$ Persei source is optically thick at $2695 \mathrm{MHz}$ when flaring, the radio emission probably comes from regions where $N_{e} \sim 10^{10} \mathrm{~cm}^{-3}$ is a good order of magnitude estimate. This then implies that $T_{e} \sim 10^{8} \mathrm{~K}, D \sim 0.3 \mathrm{AU}$, and $\theta \sim 0.01$. It is unavoidable that $\beta$ Persei will be a strong $\mathrm{X}$-ray source under these circumstances. The next most interesting of these numbers is the estimated diameter, which is exactly a factor of ten greater than the known sizes of the two stars in the close pair in the $\beta$ Persei system. It would then make the observed variable radio source of the size scale of the Roche lobe of one of the stars.

Why has $\beta$ Persei, at least, not been detected* as an X-ray source as of the time of this symposium? The most likely answer is in its transience. There is no doubt that the current flaring activity in $\beta$ Persei is considerably greater than occurred in OctoberNovember 1971 (Wade and Hjellming, 1972; Hjellming et al., 1972a), further, the work of Bolton (1972) showing the association of this flaring with optical features never before reported suggests that such activity is rare. All of this suggests that, if they exist, the Algol-type X-ray sources will be typified by transience. Indeed, transient $\mathrm{X}$-ray sources (Cen X-2, Cen X-4, Cet X-2) are known; but one cannot tell yet whether they are related to the predicted Algol-type sources.

* Several scans across the field containing $\beta$ Persei with the UHURU satellite during 1971 placed an upper limit of $10^{-4}$ the strength of Sco X-1 on the X-ray flux (Tananbaum, private communication). 


\section{Cyg X-3}

The observations (Hjellming et al., 1972b) of Cyg X-3 obtained simultaneously at 2695 and $8085 \mathrm{MHz}$ with the NRAO interferometer on May 1, 2, and 3, 1972, are shown in Figure 2. Also shown is the spectral index variation for these days. In

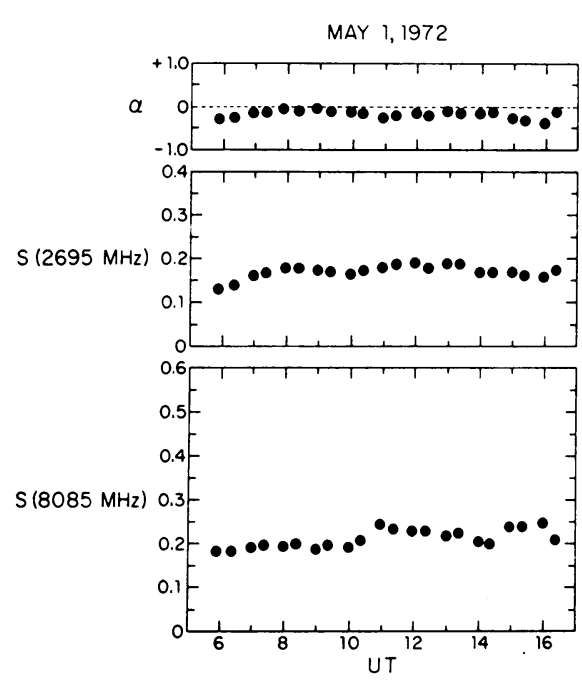

\section{CYG $X-3$}
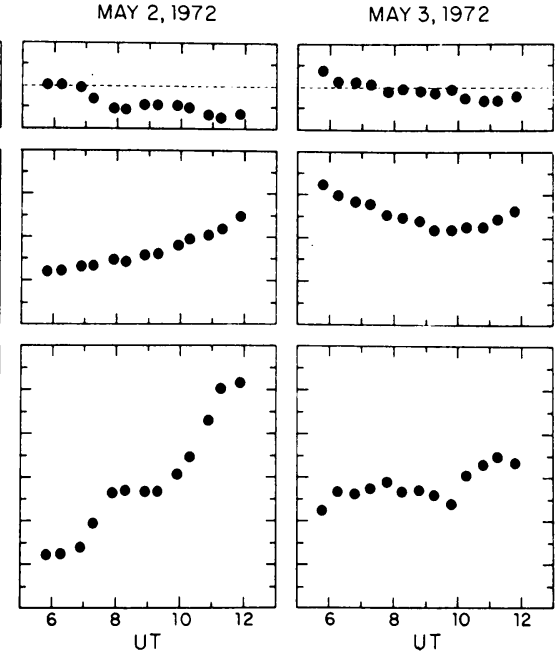

Fig. 2. The spectral index and flux densities at 2695 and $8085 \mathrm{MHz}$ are plotted as functions of UT for Cyg X-3 on May 1, 2, and 3, 1972. The error limits on the flux densities are the size of the filled circles. The flux densities are measured in flux units.

\section{$\beta$ PERSE}
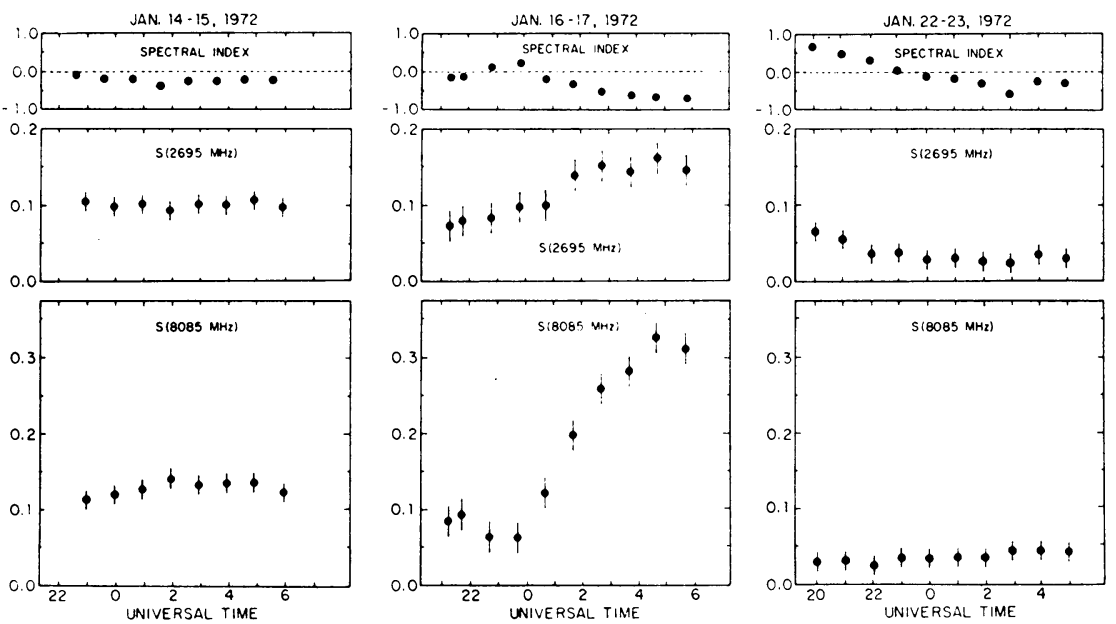

Fig. 3. The spectral index and flux densities at 2695 and $8085 \mathrm{MHz}$ are plotted as functions of UT for $\beta$ Persei (Algol) on Jan. 14/15, 16/17, and 22/23, 1972. 
Figure 3, we show the same data for $\beta$ Persei on three days which appear, in every important detail, the same as Cyg X-3 events, particularly in the spectral index variation and time scale. The Cyg X-3 flare in May 2, 1972, shows the characteristic evolution of a $\beta$ Persei flare from an optically thin thermal spectral index to an optically thick thermal spectral index. Furthermore, the non-thermal behavior of Cyg X-3 on May 3, 1972, has an exact counterpart in the only non-thermal event observed for $\beta$ Persei. It is difficult to avoid the conclusion that the radio emission mechanisms for $\beta$ Persei and Cyg X-3 are similar.

\section{Summary}

The radio data indicate that $\beta$ Persei (Algol) is a proto-type of a class of variable thermal-like radio sources of which $\beta$ Lyrae, Antares $\mathrm{B}$, and Cyg X-3 are members. It will be important to establish whether the predicted transient $\mathrm{X}$-ray emission of $\beta$ Persei exists when radio flaring occurs. All radio counterparts show evidence of both thermal and non-thermal components; with the latter being dominant in the Sco X-1 type and the former dominant in Cyg X-3 and the radio binary stars. The on-off and stability characteristics of the Cyg X-1 radio counterpart at the moment are presently unique.

\section{References}

Ables, J. G.: 1969, Astrophys. J. 155, L27.

Andrew, B. A. and Purton, C. R.: 1968, Nature 218, 855.

Bolton, C. T.: 1972, IAU Circ. No. 2388, 25 February, 1972.

Braes, L. L. E. and Miley, G. K.: 1971a, Astron. Astrophys. 14, 160.

Braes, L. L. E. and Miley, G. K.: 1971b, Nature 232, 246.

Braes, L. L. E. and Miley, G. K.: 1972a, Nature Phys. Sci. 235, 147.

Braes, L. L. E. and Miley, G. K.: 1972b, Nature, in press.

Hjellming, Z. M.: 1972, Nature, in press.

Hjellming, R. M. and Wade, C. M.: 1971a, Astrophys. J. 164, L1.

Hjellming, R. M. and Wade, C. M.: 1971 b, Astrophys. J. 168, L21.

Hjellming, R. M. and Wade, C. M.: 1971c, Astrophys. J. 168, L115.

Hjellming, R. M., Wade, C. M., Hughes, V. A., and Woodsworth, A.: 1971, Nature 234, 138.

Hjellming, R. M., Wade, C. M., and Webster, E.: 1972a, Nature 236, 43.

Hjellming, R. M., Hermann, M., and Webster, E.: 1972b, Nature, in press.

Wade, C. M. and Hjellming, R. M.: 1971a, Astrophys. J. 163, L106.

Wade, C. M. and Hjellming, R. M.: 1971b, Astrophys. J. 170, 523.

Wade, C. M. and Hjellming, R. M.: 1972, Nature 235, 270. 